\title{
FATORES DE RISCO CARDIOVASCULAR EM FEIRANTES DE UM MERCADO PÚBLICO NO SERTÃO NORDESTINO
}

CARDIOVASCULAR RISK FACTORS IN MARKET TRADERS IN A PUBLIC MARKET IN THE SEMI-ARID REGION OF THE NORTHEAST

FACTORES DE RIESGO CARDIOVASCULAR EN FERIANTES DE UN MERCADO PÚBLICO DEL SERTÃO NORDESTINO

Lucas Teixeira de Sousa Santos

Francisco Douglas Canafístula de Souza ${ }^{2}$

Ângela Maria Liberato Araújo Lopes ${ }^{3}$

Keila Maria de Azevedo Ponte Marques

Dafne Lopes Salles ${ }^{5}$ Santos LTS, Souza FDC, Lopes AMLA, Marques KMAP, Salles DL. Fatores de risco cardiovascular em feirantes de um mercado público no sertão nordestino. Sanare (Sobral, Online). $2021 ; 20(1): 25-34$

Palavras-chave: Doenças cardiovasculares; Saúde; Fatores de risco.

Keywords:

Cardiovascular Diseases; Health:

Risk Factors.

Palabras clave:

Enfermedades Cardiovasculares; Salud; Factores de riesgo.

Submetido: $24 / 04 / 2021$

Aprovado:

22/06/2021

Autor(a) para Correspondência: Lucas Teixeira de Sousa Santos E-mail: tei.lucasenfer@gmail.com

\section{RESUMO}

Este artigo objetivou identificar os fatores de risco cardiovascular de trabalhadores feirantes de um mercado público. Trata-se de uma pesquisa exploratória e descritiva realizada em fevereiro e março de $2020 \mathrm{com} 41$ feirantes no mercado público de Sobral, Ceará, aprovada pelo Comitê de Ética em Pesquisa da Universidade Estadual Vale do Acaraú (CEP/UVA). Verificouse a presença de fatores de risco na população de feirantes relacionados às características profissiográficas, nível de estresse, hábitos de alimentação e sedentarismo, tabagismo, utilização de medicamentos e falta de cuidados com a saúde, antecedentes pessoais e familiares para doenças crônicas, além de alterações nas medidas antropométricas. Concluiu-se, a partir da pesquisa, que as vulnerabilidades causadas pelo exercicio do feirante, atreladas a hábitos de vida, podem impactar negativamente a saúde cardiovascular.

1. Enfermeiro. Graduado em Enfermagem pela Universidade Estadual Vale do Acaraú (UVA).E-mail: tei.lucasenfer@ gmail.com. Orcid: https://orcid.org/0000-0001-7009-2121

2. Graduando em Enfermagem pela UVA. E-mail: douglas21091997@gmail.com.0rcid: https://orcid.org/00000002-8845-1062

3. Enfermeira. Pós-graduada em UTI geral e gestão da assistência ao paciente crítico. E-mail: angelamla1979@ gmail.com. Orcid: https://orcid.org/0000-0003-0508-0794

4. Enfermeira com Doutorado em Cuidados Clínicos em Enfermagem e Saúde pela Universidade Estadual do Ceará (UECE).E-mail: keilinhaponte@hotmail.com. Orcid: https://orcid.org/0000-0001-5215-7745

5. Enfermeira com Doutoradoem Saúde Coletiva pela UECE. E-mail: dafnelopessalles@gmail.com. Orcid: https:// orcid.org/0000-0002-8129-3428

Cert. de Redação Científica: Central das Revisões. Edição de texto: Karina Maria M. Machado. Revisão de provas: Texto definitivo validado pelos(as) autores(as). 


\section{ABSTRACT}

This article aimed to identify the cardiovascular risk factors of market traders in a public market. This is an exploratory and descriptive research carried out during February and March 2020 with 41 market traders in the public market of Sobral, Ceará, approved by the Research Ethics Committee of the Universidade Estadual Vale do Acaraú (CEP/UVA). We checked for the presence of risk factors in the population of market traders, which were related to professional characteristics, stress levels, eating habits and a sedentary lifestyle, smoking habits, the use of medications and lack of health care, personal and family history of chronic diseases, as well as changes in anthropometric measurements. From this research we concluded that the vulnerabilities caused by the market trader's exercise, linked to lifestyle habits, can negatively impact cardiovascular health.

\section{RESUMEN}

Este artículo tuvo como objetivo identificar los factores de riesgo cardiovascular de los feriantes de un mercado público. Se trata de una investigación exploratoria y descriptiva realizada en febrero y marzo de 2020 con 41 feriantes en el mercado público de Sobral, Ceará, aprobada por el Comité de Ética en Investigación de la Universidad Estatal de Vale do Acaraú (CEP / UVA). Se verificó la presencia de factores de riesgo en la población de comerciantes relacionados con características profisiográficas, nivel de estrés, hábitos alimentarios y sedentarios, tabaquismo, uso de medicamentos y falta de atención médica, antecedentes personales y familiares de enfermedades crónicas, además de cambios en las medidas antropométricas. De la investigación se concluyó que las vulnerabilidades causadas por el ejercicio del feriante, vinculadas a los hábitos de estilo de vida, pueden tener un impacto negativo en la salud cardiovascular.

\section{INTRODUÇÃO}

As doenças cardiovasculares (DVC) foram responsáveis, nos últimos anos, por cerca de $29,7 \%$ dos óbitos nacionais. Além disso, são o grupo de Doenças Crônicas Não Transmissíveis (DCNT) que mais causam mortes globais, atingindo pessoas em todos os níveis econômicos, principalmente grupos mais vulneráveis, destacando-se os de baixa renda e com baixa escolaridade ${ }^{1}$.

Existem diversos fatores de risco para 0 surgimento de DCV e a associação desses fatores causa grande prejuízo à qualidade de vida, aumentando a prevalência de adoecimento cardiovascular. Entre os riscos para a DCV, destacam-se o aumento do Índice de Massa Corporal (IMC), Circunferência de Cintura (CC), sedentarismo, estresse e fatores genéticos. Além desses fatores citados, ressaltamse também a hipertensão arterial (HAS), o uso de cigarro, a idade, a diabetes mellitus (DM) e níveis alterados de colesterol².

Vários órgãos de saúde têm estabelecido metas para a redução das DCV, junto com a sua prevenção, buscando a redução de mortes prematuras. Com isso, a prevenção e a busca de melhoria na qualidade de vida são essenciais, uma vez que o país se apresenta em desenvolvimento e possui recursos escassos ${ }^{3}$.

Devido à multifatoriedade de causas envolvendo as doenças cardiovasculares, sua elevada distribuição de fatores de risco, bem como a desigualdade entre os sexos e as condições econômicas, fazem-se necessárias investigações junto aos grupos populacionais mais expostos a esses eventos e suas complicações, buscando estratégias de intervenção mais oportunas e mais eficazes para o controle de fatores de risco, e assim reduzir seus impactos diante da saúde da população $0^{4}$.

Nesse sentido, a classe dos feirantes representa significativa parcela de uma população pouco assistida e, pela espécie de trabalho que eles realizam, estão sujeitos a vulnerabilidades de saúde ${ }^{5}$. Assim, o objetivo do estudo é identificar fatores de risco cardiovascular de trabalhadores feirantes de um mercado público.

\section{METODOLOGIA}

Trata-se de pesquisa exploratória e descritiva realizada no Mercado Público - Centro Comercial Chagas Barreto, localizado em Sobral, cidade 
situada na região Norte do Ceará, a $235 \mathrm{~km}$ de Fortaleza. 0 local do estudo conta com 280 boxes para feirantes, 60 boxes para cafezeiros e 32 pontos comerciais externos ${ }^{6}$. A escolha do ambiente ocorreu por existir uma grande concentração de pessoas leigas desassistidas de ações diretas para a promoção de saúde cardiovascular.

Foram convidados a participar do estudo os feirantes do Mercado, tendo uma amostra de 41 trabalhadores. Para serem incluídos na pesquisa precisavam ter idade a partir de 18 anos e assinar o Termo de Consentimento Livre e Esclarecido (TCLE). A pesquisa ocorreu em fevereiro e março de 2020, tendo sido interrompida a coleta de dados devido à suspensão das atividades presenciais em 17 de março de 2020 devido à pandemia causada pelo novo coronavírus.

Os participantes foram abordados individualmente nas suas barracas, sendo conscientizados sobre os objetivos e aspectos éticos da pesquisa. Posteriormente, foram convidados a assinar o TCLE e a responder ao Instrumento de Coleta de Dados contendo informações acerca do perfil sociodemográfico, deslocamento até o trabalho, características profissiográficas, nível de estresse, atividades físicas, hábitos alimentares, tabagismo, uso de drogas e medicamentos, antecedentes pessoais e familiares para doenças crônicas. Junto a isso, ocorreu a mensuração da pressão arterial, peso, altura, circunferência abdominal, frequência cardíaca e cálculo do IMC. Os dados obtidos foram organizados no software Excel 2016 e, após isso, foram elaborados gráficos e tabelas, sendo os achados discutidos de acordo com a literatura pertinente.

Este estudo está inserido em uma pesquisa guarda-chuva intitulada “Cuidando para prevenir o adoecimento cardiovascular", tendo carta de anuência aprovada pelo local da pesquisa, possuindo o parecer do Comitê de Ética em Pesquisa (CEP) com Seres Humanos da Universidade Estadual Vale do Acaraú (UVA), com n. ${ }^{\circ}$ 2.312.655/2017. Respeitaram-se os princípios da bioética conforme a Resolução n. ${ }^{0}$ 466/2012.

\section{RESULTADOS E DISCUSSÃO}

Participaram do estudo 41 feirantes. Na Tabela 1, apresenta-se o perfil dos participantes.

Tabela 1. Perfil dos participantes do estudo de acordo com dados socioeconômicos. Sobral, Ceará, 2020.

\begin{tabular}{|c|c|c|}
\hline Variáveis & Total & $\%$ \\
\hline \multicolumn{3}{|l|}{ Sexo } \\
\hline Masculino & 23 & $56 \%$ \\
\hline Feminino & 18 & $44 \%$ \\
\hline \multicolumn{3}{|l|}{ Idade } \\
\hline $20-29$ & 7 & $17 \%$ \\
\hline $30-39$ & 2 & $5 \%$ \\
\hline $40-49$ & 9 & $22 \%$ \\
\hline $50-59$ & 12 & $29 \%$ \\
\hline $60-69$ & 7 & $17 \%$ \\
\hline$>70$ & 4 & $10 \%$ \\
\hline \multicolumn{3}{|l|}{ Escolaridade } \\
\hline Analfabeto & 4 & $10 \%$ \\
\hline Ensino Fundamental Completo & 6 & $15 \%$ \\
\hline Ensino Fundamental Incompleto & 11 & $27 \%$ \\
\hline Ensino Médio Completo & 14 & $34 \%$ \\
\hline Ensino Médio Incompleto & 5 & $12 \%$ \\
\hline Ensino Superior Incompleto & 1 & $2 \%$ \\
\hline \multicolumn{3}{|l|}{ Estado Civil } \\
\hline Casado & 25 & $61 \%$ \\
\hline Divorciado & 2 & $5 \%$ \\
\hline Solteiro & 11 & $7 \%$ \\
\hline Viúvo & 3 & $27 \%$ \\
\hline
\end{tabular}




\begin{tabular}{|c|c|c|}
\hline Variáveis & Total & $\%$ \\
\hline \multicolumn{3}{|l|}{ Raça } \\
\hline Branca & 4 & $17 \%$ \\
\hline Negra & 3 & $12 \%$ \\
\hline Parda & 17 & $71 \%$ \\
\hline Total de participantes & 41 & $100 \%$ \\
\hline
\end{tabular}

Fonte: Dados da pesquisa.

Como verificado, $56 \%$ (23) do público é masculino, mas há parcela quase igualitária entre público feminino, com diferença de $6 \%$. A profissão de feirante, que antes era entendida como própria do sexo masculino, vem sendo adotada por mulheres, devido às mudanças histórico-culturais que as colocam à frente da jornada de trabalho, em busca da sua própria renda e sustento ${ }^{5}$.

Quanto à idade desses trabalhadores, houve um predomínio entre as idades de 50 a 59 anos, mas há grande variação de faixas etárias. Outros estudos realizados com feirantes mostraram que a faixa etária encontrada nessa categoria é variada ${ }^{5-8}$. Em relação à escolaridade, há presença de diversos níveis, mas predomínio dos que completaram o ensino médio. Outras características presentes são a predominância de casados e, quanto à cor, pardos se mostraram em maior quantidade. Dessa forma, o perfil se apresenta com diversidade, podendo ser levadas em conta as características da profissão, por ser informal e autônoma.

Os fatores de riscos cardiovasculares investigados após a coleta das variáveis sociodemográficas foram analisados nos seguintes aspectos: meios de locomoção para o trabalho, características profissiográficas, nível de estresse, realização de atividades físicas, hábitos alimentares, tabagismo, uso de drogas e medicamentos, histórico pessoal e familiar para doenças crônicas e dados antropométricos.

Quando questionados sobre o deslocamento até o trabalho, obteve-se que $68 \%$ (28) dos feirantes possuíam transporte próprio. Entre os transportes citados, estão: moto - $64 \%$ (18), bicicleta $22 \%$ (6) e carro - 14\% (4). Apenas 32\% (13) não possuíam transporte próprio, locomovendo-se para o trabalho por mototáxi - 46\% (6), caminhada $23 \%$ (3), topique/ônibus - 16\% (2) e carona - 15\% (2).

Segundo as análises da Vigilância de Fatores de Risco e Proteção para Doenças Crônicas por Inquérito Telefônico (Vigitel), do Sistema de
Vigilância de Fatores de Risco de DCNT do Ministério da Saúde, que contabiliza, em pelo menos 30 minutos diários, a forma de ida ao trabalho como marcador para atividade física, apenas $22 \%$ dos feirantes se apresentam ativos no deslocamento até o local de trabalho, sendo os que se deslocam de bicicleta, $15 \%(6)$, e os que vão caminhando, $7 \%$ (3), sobrepondo-se aos $14,1 \%$ das cidades consultadas pelo sistema ${ }^{9}$.

Para o tempo de trabalho, 37\% (13) dos feirantes dedicam-se ao labor de cinco a nove horas por dia, $56 \%$ (15) trabalham de dez a 12 horas por dia, outros $7 \%$ (3) trabalham mais de 12 horas por dia. Com as respostas é notável a sobrecarga de horas de trabalho, de modo que muitos relataram que começavam a trabalhar na madrugada e iam até as quatro da tarde, horário de fechamento do mercado, acumulando mais de $12 \mathrm{~h}$ de trabalho ao dia. A carga horária trabalhada pelos feirantes da pesquisa é análoga a um estudo encontrado na literatura, em que os feirantes iniciavam as atividades geralmente às $3 \mathrm{~h}$ da manhã e enceravam às $15 \mathrm{~h}$ ou $16 \mathrm{~h}$ da tarde ${ }^{10}$. Tal fato também se encontra em outro estudo, no qual a jornada de trabalho varia entre seis e 13 horas por dia ${ }^{5}$.

Entre os fatores de riscos para acidentes de trabalho entre trabalhadores informais está 0 esforço físico ${ }^{11}$. Nesse sentido, 68\% (28) dos participantes referiram o levantamento de peso como fator presente no trabalho, devido ao descarregamento de caixas. Outro fator de risco é o estresse, que tem caráter crônico com capacidade de causar diversos danos ao organismo, provocando disfunções e enfermidades ${ }^{12}$. Na avaliação, 5\% (2) revelou ter muito pouco estresse, $39 \%$ (16) refere pouco estresse, $29 \%$ (12) tem estresse moderado e $27 \%$ (11), estresse excessivo.

Para as práticas físicas, $49 \%$ (20) diz realizar atividades físicas e $51 \%$ (21) tem características sedentárias. Entre as atividades físicas realizadas pelos $49 \%$ está a caminhada, a qual é praticada por $65 \%$ (13) dos sujeitos da pesquisa; a hidroginástica, $15 \%$ (3); o pedalo, 10\% (2); a capoeira, 5\% (1); e 
a musculação, 5\% (1). Para a quantidade de vezes em que praticam atividades físicas, $35 \%$ (7) dos entrevistados praticam cinco vezes/semana, outros $35 \%$ (7) praticam três vezes/semana, 25\% (5) praticam quatro vezes/semana e $5 \%$ (1) pratica seis vezes/semana.

0 ato de praticar exercícios traz benefícios à saúde, dentre eles: a redução de complicações crônicas, levando à menor possibilidade de morte prematura por doenças crônicas, além de outras patologias desencadeadas por sedentarismo e obesidade ${ }^{13}$. Cerca de 28,1 milhões de brasileiros praticam atividade física, sendo a atividade mais realizada a caminhada, que acumula um total de 13,8 milhões de adeptos ${ }^{14}$.

Um dos fatores de risco mais prevalentes para doenças cardiovasculares é o sedentarismo ${ }^{15}$. No Brasil, o Instituto Brasileiro de Geografia e Estatística (IBGE) aponta um total de 100,5 milhões de pessoas sem realizar atividades físicas ou praticar esportes, e, entre as regiões, o Nordeste se destaca com a maior taxa de inativos para atividades físicas, com $63,7 \%$, em consonância com $51 \%$ dos feirantes não praticantes ${ }^{14}$.

Uma alimentação errada ou com exageros acarreta problemas à saúde da população. Maiores implicações surgem de alimentações gordurosas, com excesso de carboidratos, açúcares e sódio. Tais alimentos exercem malefícios sobre a pressão arterial, coração, rins e sistema venoso $\operatorname{arterial}^{16}$. Em relação ao consumo alimentar, os feirantes relataram dificuldade para manter uma alimentação regular e saudável, por passarem a maior parte do dia no mercado cuidando de suas bancas, impossibilitados de irem para casa em horário de almoço, o que thes compeliam a alimentar-se de comida comprada pelo centro da cidade, e outros levavam o almoço feito do dia anterior.

Assim, encontrou-se a seguinte ordem de alimentos consumidos diariamente, de forma decrescente: frutas - 65\% (26), cereais $-59 \%(24)$, vegetais $-44 \%$ (18), laticínios - 34\% (14), carne - 27\% (11) e doces - 10\% (4). Quanto ao peixe, a maior taxa de consumo é de forma mensal, sendo consumido por $54 \%$ (22) de uma a quatro vezes por mês.

Nessa perspectiva, os alimentos que mais devem ser consumidos são os advindos de fontes naturais, como frutas e verduras, seguidos do consumo moderado de alimentos com maiores taxas de calorias e gorduras, como pães, macarrão, tubérculos, farinhas e óleos vegetais, e os alimentos que devem ser consumidos em menor quantidade, como carnes, manteigas, queijos brancos e amarelos, entre outros ${ }^{17}$. Assim, levando em consideração os alimentos mais consumidos diariamente pelos feirantes, há uma inversão no consumo de cereais e vegetais.

Ao serem questionados sobre hábitos de tabagismo, constatou-se que $66 \%$ (27) nunca havia fumado, 32\% (13) é ex-fumante e apenas $2 \%$ (1) é fumante ativo. Nos últimos anos, no Brasil, ocorreu o declínio do uso de tabaco, uma redução de 19\%, tornando-se um dos países com a mais baixa taxa de consumo do mundo ${ }^{18}$. A medida mais eficaz como prevenção para doenças provocadas pelo uso de tabaco é a interrupção do ato de fumar, pois, além das complicações cardiovasculares e cancerígenas, tabagistas têm em média cinco anos de vida a menos do que não fumantes ${ }^{19}$. Portanto, 0 ato de cessar o uso de cigarros é uma ação positiva para os feirantes, devido à influência que outros fatores de risco exerceriam sobre a saúde, somados ao uso de tabaco.

Já para o consumo de álcool, $88 \%$ (5) disse não consumir e $12 \%$ (5) disse fazer uso apenas aos finais de semana, por conta do trabalho. 0s efeitos do consumo de álcool são uma linha tênue entre a moderação, podendo ser associados a contribuições benéficas para a saúde cardiovascular, ou não, caso haja exagero ${ }^{20}$.

Referente a consultas médicas regulares, $56 \%$ (23) deles afirmaram não ir ao médico com frequência, $22 \%$ (9) dizem que vão quando doentes e outros $22 \%$ (9) vão com frequência. A justificativa mais apontada para a lacuna de cuidados com a saúde é a falta de tempo pelas horas trabalhadas, o que causa inviabilidade de acesso às unidades de saúde por feirantes, pela influência da extensa jornada de trabalho e o desconhecimento sobre o estado de saúde ${ }^{10}$.

Para o uso de medicamentos com prescrição médica, 39\% (16) deles fazem o uso; 61\% (25), não. Entre as classes das medicações em uso estão os anti-hipertensivos, com $44 \%$ (12) e os antidiabéticos, $30 \%$ (8); sendo que $26 \%$ (7) faz uso de outros tipos de medicamentos. Entre os remédios mencionados, identificaram-se: losartana e metformina, pertencentes à classe dos medicamentos mais utilizados pelos feirantes, a sinvastatina, enalapril, ácido acetil salicílico, anticoncepcional, bombinha para asma, captopril, 
glibenclamida, hidroclorotiazida e vertix. A classe de drogas mais utilizadas pelos feirantes é a dos antihipertensivos e os antidiabéticos, classe de medicamentos pertencentes às duas das substâncias mais comercializadas no Brasil, a losartana potássica e o cloridrato de metformina, em terceiro e segundo lugar, respectivamente ${ }^{21}$.

Em relação ao uso de remédios sem prescrição, 27\% (11) dos feirantes afirmam utilizar e $73 \%$ (30) negam a utilização. As medicações utilizadas sem prescrição pertencem à classe de analgésicos - 64\% (7) e antiinflamatórios - 36\% (4). 0 grupo terapêutico mais utilizado sem prescrição pelos brasileiros é o de analgésicos, seguido dos relaxantes musculares e anti-inflamatórios, com maior prevalência de automedicação na região Nordeste 22 .

Como se sabe, entre os fatores de risco para doenças cardiovasculares estão a hipertensão, a diabetes e a dislipidemia, desencadeadora da aterosclerose. Assim, a prevalência dessas doenças nos feirantes também foi investigada. Entre eles, 68\% (28) tinham hipertensão; 22\% (9), diabetes mellitus; e 24\% (10), dislipidemia. Corroborando a Pesquisa Nacional de Saúde, a doença crônica que mais se destaca na população brasileira é a HAS e a quinta mais presente é a diabetes ${ }^{23}$. No que tange à dislipidemia, análise de níveis séricos da população brasileira aponta que cerca de um terço dos adultos apresenta alterações no colesterol ${ }^{24}$.

Quanto ao histórico familiar para doenças crônicas, há presença de hipertensão na família de $61 \%$ (25) dos feirantes, mais presente nas mães e irmãs, com 35\% (13) e $23 \%$ (8), respectivamente; outros parentes são pai - 14\% (5), avó - 11\% (4), irmão - 9\% (3) e tia - 6\% (2). Já para diabetes, $46 \%$ tem histórico familiar, sendo mais presente em irmãs, com 29\% (6), e em mãe e pai, com 19\% (4) cada; outros familiares são irmão - $14 \%$ (3), esposa e avó, com $5 \%$ (1) cada.

Tais resultados têm semelhanças com os apontados em estudo sobre fatores de risco para doenças crônicas, apresentando maior prevalência de antecedentes familiares para hipertensão e diabetes ${ }^{25}$. Segundo estudo que avaliou a prevalência de hipertensão e diabetes autorreferida na população brasileira, há maior prevalência de mulheres do que de homens acometidos ${ }^{24}$.

Para Doença Aterosclerótica Coronariana, 37\% (15) dos pesquisados afirmaram ter parentes acometidos, com maior incidência para mãe, com 47\% (7), e avó, com $27 \%$ (4); outros familiares são irmã e pai, com $13 \%$ (2) cada.

Diversos agravos à saúde foram impulsionados pela industrialização, tecnologias, urbanização e estilos de vida, ligados diretamente às afecções cardiovasculares. Tais agravos podem ser identificados com técnicas simples que determinam riscos, entre elas estão as medidas antropométricas ${ }^{26}$. Nesse sentido, avaliaram-se as seguintes medidas antropométricas e níveis pressóricos dos feirantes, organizados na Tabela 2:

Tabela 2. Distribuição dos participantes da pesquisa de acordo com as Medidas Antropométricas. Sobral, Ceará, 2020.

\begin{tabular}{l|l|l}
\multicolumn{1}{c|}{ Medidas } & Total & $\%$ \\
\hline Circunferência da Cintura & & \\
\hline $\begin{array}{l}\text { Maior risco para DCV } \\
\text { Menor risco para DCV }\end{array}$ & 10 & $76 \%$ \\
İndice de Massa Corporal & & $24 \%$ \\
\hline $\begin{array}{l}\text { Adequado } \\
\text { Baixo Peso }\end{array}$ & 14 & $34 \%$ \\
Sobrepeso & 3 & $7 \%$ \\
Obesidade & 13 & $32 \%$ \\
Relação Cintura Quadril & 11 & $27 \%$ \\
\hline Maior risco para DCV & & \\
Menor risco para DCV & 36 & $88 \%$ \\
\hline
\end{tabular}




\begin{tabular}{l|ll}
\multicolumn{1}{c|}{ Medidas } & Total & $\%$ \\
\hline \hline Pressão Arterial & & \\
\hline Normal & 22 & $54 \%$ \\
Pré-hipertensão & 8 & $20 \%$ \\
Estágio I & 7 & $17 \%$ \\
Estágio II & 3 & $7 \%$ \\
Estágio III & 1 & $2 \%$ \\
\hline
\end{tabular}

Fonte: Dados da pesquisa.

Para analisar as medidas de Circunferência da Cintura, utilizou-se como ideal para homens o valor de $\geq 94,0$ $\mathrm{cm}$ e para mulheres, $\geq 80,0 \mathrm{~cm}$. Assim, $76 \%$ dos feirantes apresentaram CC elevada. Entre as mulheres, apenas $22 \%$ (4) se mostraram com valores adequados e 78\% (14) demonstraram alterações na CC. Quanto aos homens, $73 \%$ (17) apresentaram alterações e $26 \%$ (6) estavam no padrão normal.

Quanto aos Índices de Massa Corporal, calcularam-se os dos feirantes de 20 a 59 anos pelo padrão da Organização Mundial da Saúde (OMS), que define como obesos pessoas com IMC $\geq 30$. Para o IMC de pessoas acima de 60 anos é adotado um ponto de corte diferente, levando em consideração as alterações fisiológicas que ocorrem com a senescência: idosos com IMC $\leq 22$ são classificados como baixo peso, $<27$ têm peso adequado e com $\geq 27$ são classificados como sobrepeso ${ }^{9}$. Conforme os cálculos, $32 \%$ dos pesquisados tiveram IMC para sobrepeso e $27 \%$ para obesidade, ou seja, mais de $50 \%$ dos feirantes apresentaram alterações de peso.

De forma ampla, dados da Vigitel mostram que em 27 cidades brasileiras há predominância de pessoas com excesso de peso $(55,7 \%)$ do que com obesidade $(18,8 \%)^{9}$. A população que manifesta sobrepeso e obesidade e que tem maior localização de gordura na região abdominal está mais associada às doenças como hipertensão arterial sistêmica, diabetes e dislipidemia ${ }^{27}$.

A Relação Cintura Quadril (RCQ) também é uma medida calculada para avaliar o risco de doenças cardiovasculares, de modo que o índice de corte para definir o risco em mulheres é menor ou igual a 0,80 e para homens é $0,90^{\circ}$. Nesses padrões, $88 \%$ apresentou um maior risco para DCV. Para 78\% (14) das mulheres, a RCQ apresentou-se alterada e em apenas 22\% (4) estava normal. Já para os homens, 74\% (17) demonstraram alterações e $26 \%$ (6), não. Pesquisa realizada no Paraná, com 60 mulheres, afirma que apenas uma das mulheres não apresentou alterações na $\mathrm{RCQ}^{28}$.

Quanto às medidas pressóricas, foi adotada como base a $7^{\text {a }}$ Diretriz Brasileira de Hipertensão para a classificação ${ }^{29}$. A Diretriz estabelece os seguintes valores, mostrados na Tabela 3:

Tabela 3. Classificação da Pressão Arterial de acordo com medição casual. Sobral, Ceará, 2020.

\begin{tabular}{lcc} 
Classificação & $\begin{array}{c}\text { Pressão } \\
\text { Arterial Sistólica } \\
(\mathbf{m m ~ H g})\end{array}$ & $\begin{array}{c}\text { Pressão Arterial Diastólica } \\
(\mathbf{m m H})\end{array}$ \\
\hline Normal & $\leq 120$ & $\leq 80$ \\
Pré-hipertensão & $121-139$ & $81-89$ \\
Hipertensão Estágio I & $140-159$ & $90-99$ \\
Hipertensão Estágio II & $160-179$ & $100-109$ \\
Hipertensão Estágio III & $\geq 180$ & $\geq 110$ \\
\hline
\end{tabular}

Fonte: $7^{\text {a }}$ Diretriz Brasileira de Hipertensão ${ }^{29}$.

Quanto às alterações, $20 \%$ (8) apresentou medidas pressóricas para pré-hipertensão, 17\% (7) para hipertensão em estágio I, 7\% (3) para estágio II e apenas 2\% (1) estava enquadrado no estágio III; somando as modificações, 46\% (19) apresenta pressão arterial alterada. Para os homens, 48\% (11) apresentaram PA alterada, e 52\% (12), normal. Já entre as mulheres, 44\% (8) tiveram alterações pressóricas e 56\% (10), não.

Conforme apresentado, os feirantes dispõem de fatores significativos para favorecer o adoecimento cardiovascular, intrínsecos ao seu próprio trabalho, ligados aos seus hábitos e à sua própria genética e organismo, que, associados entre si, podem acarretar implicações à saúde de forma ampla. Assim, faz-se necessária a prática de intervenções eficazes para disseminar informações acerca do adoecimento cardiovascular, de forma 
a colocar os feirantes, ativamente, à frente do seu autocuidado à medida que dúvidas forem sendo esclarecidas e conhecimentos sendo adquiridos ${ }^{30}$.

\section{CONCLUSÃO}

A investigação dos fatores de risco cardiovascular dos feirantes possibilitou visualizar a vulnerabilidade a que essa classe está inserida. Os riscos estão relacionados ao próprio exercício de trabalho, atrelados aos hábitos e estilo de vida. Muitos se encontram expostos a riscos para a saúde que parecem ser agravados pelas particularidades do labor: a alta carga horária; a necessidade de estar muito cedo no mercado para receber mercadoria, desregulando o sono; a alimentação saudável que, para uns, é impossibilitada por passarem a maior parte do dia na feira; além de alguns não possuírem transporte próprio para facilitar o translado entre casa e trabalho, tendo que pagar para realizar a ação. A alta taxa de horas trabalhadas também influencia na baixa procura médica para cuidados de rotina, por falta de tempo, fazendo com que essas consultas ocorram apenas quando há adoecimento.

0 grupo de agravos mencionado, juntamente com as alterações nas medidas antropométricas, juntos, têm grande potencial para impactar na saúde cardiovascular dessa população. Pelas doenças cardiovasculares serem, muitas vezes, silenciosas, deixando os acometidos ainda mais vulneráveis, torna-se importante e relevante 0 incentivo a outras pesquisas para melhorar a qualidade de vida dessa classe de trabalhadores, com foco nas suas condições de trabalho.

Entre os pontos negativos da pesquisa, observase a baixa quantidade de participantes, por consequência da interrupção da coleta de dados devido à pandemia. Também se destaca o desafio da inserção dos pesquisadores dentro do ambiente e horário de trabalho dessas pessoas, o que afetou em alguns momentos a linearidade da pesquisa, por conta de interrupção de clientes e barulhos.

\section{CONTRIBUIÇÃO DOS AUTORES}

Lucas Teixeira de Sousa Santos contribuiu com o delineamento, a realização da pesquisa e a redação do manuscrito. Francisco Douglas Canafístula de Souza contribuiu com a realização da pesquisa e a redação do manuscrito. Ângela Maria Liberato Araújo contribuiu com a realização da pesquisa. Keila Maria de Azevedo Ponte Marques contribuiu com o delineamento, redação e a revisão crítica do manuscrito. Dafne Lopes Salles contribuiu com a redação e a revisão crítica do manuscrito.

\section{REFERÊNCIAS}

1. Malta DC, Andrade SSCA, Oliveira TP, Moura L, Prado RR, Souza MFM. Probabilidade de morte prematura por doenças crônicas não transmissiveis, Brasil e regiões, projeções para 2025. Rev bras epidemiol [serial on the internet]. 2019 [cited 2020 May 25];22(1):1-13. Available from: https://www.scielo.br/j/rbepid/a/ r7QkT4hR3HmkWrBwZc6bshG/abstract/?lang=pt

2. Massaroli LC, Santos LC, Carvalho GG, Carneiro SAJF, Rezende LF. Qualidade de vida e o IMC alto como fator de risco para doenças cardiovasculares: revisão sistemática. Revista da Universidade Vale do Rio Verde [serial on the internet]. 2018 [cited 2020 May 20];16(1):1-10. Available from: http://periodicos.unincor.br/index .php/ revista unincor/article/view/3733/pdf 794

3. Siqueira ASE, Siqueira-Filho AG, Land MGP. Análise do Impacto Econômico das Doenças Cardiovasculares nos Últimos Cinco Anos no Brasil. Arq Bras Cardiol [serial on the internet]. 2017 [cited 2020 Jan 17];1(109):39-46. Available from: $\quad$ https://www.scielo.br/j/abc/a/TjBM VD 83 F 7 NMGNCJsP9kXKD/? l a n g $=p t$

4. Lunkes LC, Murgas LDS, Dorneles EMS, Rocha CCMBM, Machado GJ. Fatores Socioeconômicos relacionados às doenças cardiovasculares: uma revisão. Hygeia [serial on the internet]. 2018 [cited 2019 0ct 10];28(14):5061. Available from: http://www.seer.ufu. br/index.php/hygeia/article/view/40663

5. Lima KS, Almeida AM. 0 conhecimento de feirantes sobre a hipertensão arterial e suas complicações. Rev. baiana saúde pública [serial on the internet]. 2014 [cited 2020 Mar 17]; 38(4):865-81. Available from: http://files.bvs. br/upload/S/0100-0233/2014/v38n4/a4914.pdf

6. Sobral. Prefeitura de Sobral: Mercado Público [home-page on the internet]. [cited 2020 Jan 15]. Available from: http://stde. sobral.ce.gov.br/mercado-publico

7. Vale PRLE, Prata DRA, Amorim RC, Aguiar MGG. Arranjos familiares: modos de conviver com o adoecimento de famílias de feirantes. Rev Bras Ciên Saúde [serial on the internet]. 2017 [cited 2020 Mar 18];21(4):347-454. 
Available from: https://pesquisa.bvsalud.org/ portal/resource/pt/biblio-1007142

8. Vale PRLF, Santos TP, Saturtino MN, Aguiar MGA, Carvalho ESS. Itinerários terapêuticos de feirantes diante das necessidades de saúde dos familiares. Rev baiana enferm [serial on the internet]. 2015 [cited 2020 June 01];29(4):372-81. Available from: $\quad$ https://periodicos.ufba.br/index.php/ enfermagem/article/view/13396

9. Brasil. Vigitel Brasil 2019: vigilância de fatores de risco e proteção para doenças crônicas por inquérito telefônico [home-page on the internet]. [cited 2020 May 18]. Available from: http://www. crn1.org.br/wp-content/uploads/2020/04/vigitelbrasil-2019-vigilancia-fatores-risco.pdf?×53725

10. Tavares APM, Silva AK0, Fernandes MA. Planejamento estratégico situacional e a aplicabilidade à saúde do trabalhador: um estudo com feirantes. Rev Enferm UFPI [serial on the internet]. 2016 [cited 2020 May 22];5(3):7275. Available from: https://revistas.ufpi.br/ index.php/reufpi/article/view/1457/pdf

11. Rios MA, Nery AA, Rios PAA, Casotti CA, Cardoso JP. Fatores associados a acidentes de trabalho envolvendo trabalhadores informais do comércio. Cad Saúde Pública [serial on the internet]. 2015 [cited 2020 May 30];31(6):1199-1212. Available from: https://www.scielosp.org/pdf/csp/2015. v31n6/1199-1212/pt

12. Prado CEP. Estresse ocupacional: causas e consequencias. Rev Bras Med [serial on the internet]. 2016 [cited 2020 May 31];14(3):285-89. Available from: https://www.rbmt.org.br/details/ $122 / \mathrm{pt}-\mathrm{BR} / \mathrm{estresse-ocupacional- \text {causas-e- }}$ consequências

13. Santos G0, Ceolin V, Dias JA. Exercícios físicos e os benefícios em adultos. In: Silivinski CT, editor. Análise crítica das ciências da saúde 4. Ponta grossa: Atena editora; 2020. Available from: https://www.finersistemas.com/atenaeditora/ index.php/admin/api/artigoPDF/23882

14. Instituto Brasileiro de Geografia e Estatísticas. Práticas de esporte e atividade física: 2015 [home-page on the Internet]. [cited 2020 May 31]. Available from: https://biblioteca.ibge.gov.br/ index.php/biblioteca-catalogo id $=2100364$ \&iew $=$ detalhes

15. Cardoso FN, Domingues TAM, Silva SD, Lopes JL. Fatores de risco cardiovascular modificáveis em pacientes com hipertensão arterial sistêmica. Rev Min Enferm [serial on the internet]. 2020 [cited 2020 May 30];24(1275):1-8. Available from: https://pesquisa.bvsalud.org/portal/resource/pt/ biblio-1051265
16. Bankoff ADP, Bispo IM0, Souza MAB. Estudo da cultura alimentar, hábitos de vida e influências sobre as doenças crônicas não transmissiveis. Rev Saúde e Meio Ambiente [serial on the internet]. 2020 [cited 2020 June 01];10(1):118. Available from: https://periodicos.ufms. br/index.php/sameamb/article/view/9118

17. Brasil. Alimentação Cardioprotetora: manual de orientações para profissionais de saúde da Atenção Básica [document on the internet]. Brasília (DF); 2018 [cited 2020 May 21]. Available from: http://bvsms.saude.gov.br/bvs/publicacoes/ alimentacao cardioprotetora.pdf

18. Malta DC, Vieira ML, Szwarcwald CL, Caixeta $R$, Brito SMF, Reis AACD. Tendência de fumantes na população Brasileira segundo a Pesquisa Nacional de Amostra de Domicílios 2008 e a Pesquisa Nacional de Saúde 2013. Rev bras epidemiol [serial on the internet]. 2015 [cited 2020 June 03];18(2):25-56. Available form: https://www.scielo.br/j/rbepid/a/WKdynVQzFT $\mathrm{dwj \times 57WmHQ5tn/ \text {?lang } = p t}$

19. Oliveira GMM, Mallet ALR. Tabagismo. In: Rocha RM, Martins WA, editores. Manual de Prevenção Cardiovascular. Rio de Janeiro: SOCERJ; 2017.

20. Pitito BA, Moraes ACF, Ferreira SRG. 0 lado saudável do consumo de bebida alcoólica. Rev USP [serial on the internet]. 2012 [cited 2020 May 15];1(96):55-68. Available from: http://www. revistas.usp.br/revusp/article/view/52257

21. Agencia Nacional de Vigilância Sanitária. Anuário estatístico do mercado farmacêutico 2018 [home-page on the internet]. Brasilia: Anvisa; 2019. [cited 2020 June 03]. Available from: http:// portal.anvisa.gov.br/documents/374947/3413536/ Anu $\%$ C $3 \%$ A1rio+Estat $\%$ C $3 \%$ ADstico + do + Mercado $+F$ armac\%C3\%AAutico+-+2018/c24aacbf-4d0c-46a7bb86-b92c170c83e1

22. Arrais PSD, Fernandes MEP, Dal Pizzol TS, Ramos LR, Mengue SS, Luiza VL. Prevalência da automedicação no Brasil e fatores associados. Rev Saúde Pública [serial on the internet]. 2016 [cited 2020 June 03];2(50):1-13. Available from: $\quad$ www.scielosp.org/article $/ r$ sp/2016.v50suppl2/13s/pt/

23. Malta DC, Stopa SR, Szwarcwald CL, Gomes NL, Silva Júnior JB, Reis AAC. A vigilância e 0 monitoramento das principais doenças crônicas não transmissíveis no Brasil: Pesquisa Nacional de Saúde 2013. Rev bras epidemiol [serial on the internet]. 2015 [cited 2020 May 25];18(2):3-16. Available from: https://www.scielo.br/pdf/rbepid/ v18s2/1980-5497-rbepid-18-s2-00003.pdf

24. Malta DC, Szwarcwald CL, Machado IE, Pereira 
CA, Figueiredo AW, Nogueira de Sá ACMG, et al. Prevalência de colesterol total e frações alterados na população adulta brasileira: Pesquisa Nacional de Saúde. Rev bras epidemiol [serial on the internet]. 2019 [cited 2020 June 03];22(2):1-13. Available from: https://www.scielo.br/j/rbepid/a/ gxFK6KvfqFRPWJxwJKmhFqq/?lang=pt

25. Brevidelli MM. Fatores de risco para doenças crônicas entre participantes do programa Vivendo com Estilo e Saúde. 0 Mundo da Saúde [serial on the internet]. 2017 [cited 2020 June 03];41(4):606-16. Available from: https:// bvsms.saude.gov.br/bvs/periodicos/mundo saude artigos/fatores doenca cronica.pdf

26. Pohl HH, Arnold EF, Cerentini TM, Reuter EM, Reckziegel MB. Indicadores antropométricos e fatores de risco cardiovascular em trabalhadores rurais. Rev Bras Med Esporte [serial on the internet]. 2018 [cited 2020 June 01];24(1):64-68. Available from: https://www.scielo.br/j/rbme/ a/7ZXzSGKMvcy9FFRbLmcwNbG/abstract/?lang=pt

27. Barroso TA, Marins LB, Alves R, Gonçalvez ACS, Barroso SG, Rocha GS. Associação entre a obesidade central e a incidência de doenças e fatores de risco cardiovascular. Int J Cardiovasc Sci [serial on the internet]. 2017 [cited 2020 May 13];30(5):41624. Available from: http://www.onlineijcs.org/ sumario/30/30-5/original6.asp

28. Vieira RS, Gallo AM, Carvalho CJA, Araujo JP, Cabral R, Eler GJ. Fatores de risco para desenvolvimento de doença cardiovascular em mulheres. Invest Educ Enferm [serial on the internet]. 2017 [cited 2020 June 05];35(1):3545. Available from: https://revistas.udea.edu.co /index.php/iee/article/download/327343/2078704 $\underline{6}$ ? inline $=1$

29. Malachias MVB, Souza WKSB, Plavnik FL, Rodrigues CIS, Brandão AA, Neves MFT, et al. $7^{\text {a }}$ Diretriz Brasileira de Hipertensão Arterial. Arq Bras Cardiol [serial on the internet]. 2016 [cited 2020 June 05];2016;105(3):1-83. Available from: $\quad$ http://publicacoes.cardiol.br/2014/ diretrizes/2016/05 HIPERTENSA0 ARTERIAL.pdf

30. Braga YKB, Vieira RBS, Cardoso MAP, Frota KC, Ponte KMA. Saúde cardiovascular: saber de alunos e funcionários de uma universidade pública. Sanare (Sobral, Online) [serial on the internet]. 2019 [cited 2021 June 22];19(2):2431. Available from: https://sanare.emnuvens .com.br/sanare/article/view/1472
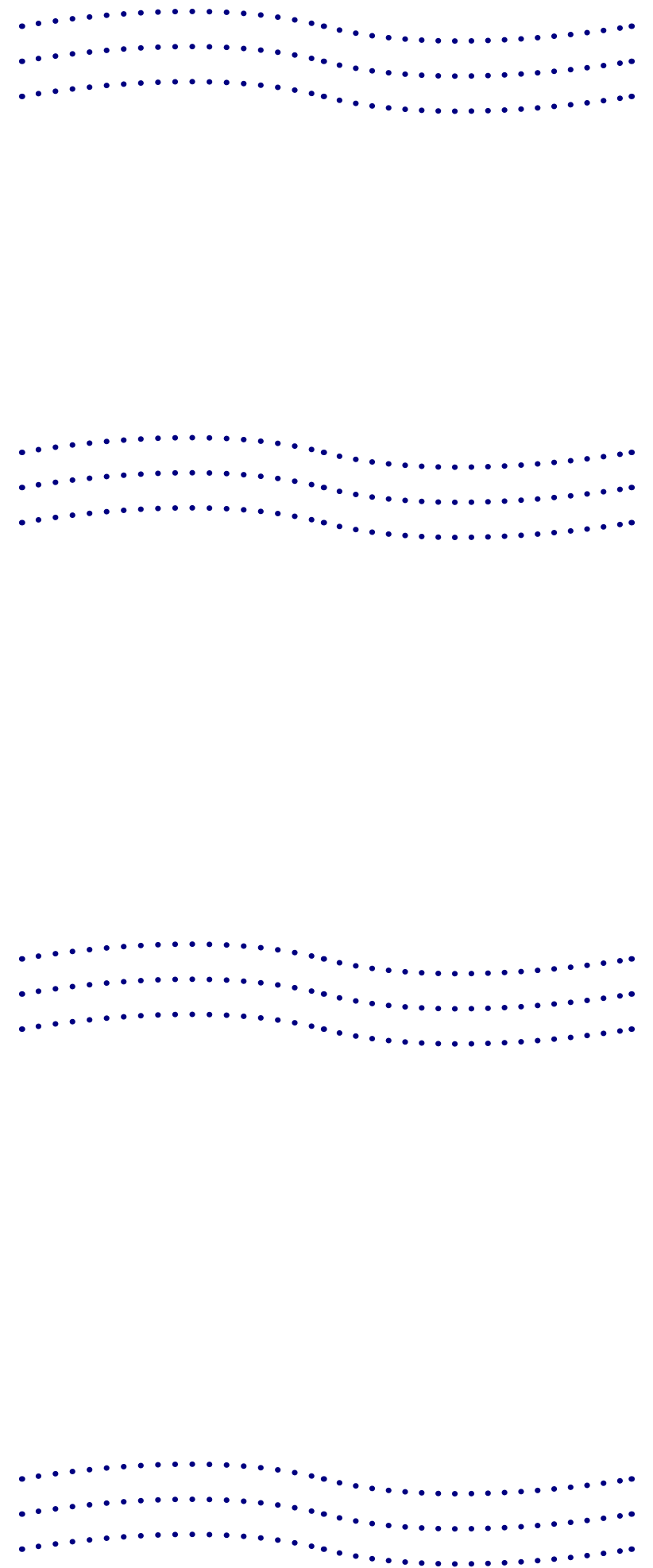